\title{
Fluid damping phenomena in a slender microbeam modelled on nonclassical theory
}

\author{
P. Belardinelli ${ }^{\text {a }}$, S. Lenci, and G. Cocchi \\ DICEA, Polytechnic University of Marche, 60131 Ancona, Italy
}

\begin{abstract}
This work deals with the evaluation of the squeeze-film damping in an electrically-actuated microbeam considering the effects of an imposed static deflection. The model presents a reliable modelling of the mechanical behaviour by improving the classical approach with the features of the strain-gradient elasticity theory. Taking into account a correction of the electric actuation for the fringing field effects, a parametric analysis is performed. The work pays attention to evaluate the damping force on the beam surface both in small static deflection regime and near the static pull-in. The results show that the correction for the finiteness of beam edges and the high-order material parameters affect the response only at large deflections. A brief study on the static behaviour is carried out highlighting how the response is affected by the strain-gradient elasticity theory. A parametric analysis of the damping force is presented and the properties of the cut-off point are studied.
\end{abstract}

\section{Introduction}

Due to the numerous problems and the large number of engineering fields involved, the interest in Micro-Electrical Mechanical-Systems (MEMS) is continuously growing. Several applications of these devices as sensors $[1,2]$ require a thorough analysis of loss mechanisms. Unfortunately one of the main difficulties in the modeling is related to the multiphysics nature of the system [3]. The phenomena of damping in microstructures are widely studied [4], in [5] a model for the fluid damping in parallel surfaces moving perpendicularly is developed. The work of [6] reports an overview and progress of research on squeeze film air damping, [7] uses a perturbation method to study the fluid damping in microplates.

This work presents an application of the model developed by [8] where the effect of the static deflection is analysed. As explained in [9], the static behaviour depends on the competition between the mechanical restoring force of the microbeam and the opposing electrostatic force. The structural instability phenomenon of pull-in is the collapse of the structure due to the too high voltage. The mechanical governing equation, based on [10], uses the modified strain-gradient elasticity proposed by [11] to take into account local properties of the microstructure. The analysed microbeam bends under an electric force caused by an applied voltage difference. A correction for the fringing field effects is introduced in the one-dimensional model to account for the finite actual dimensions of the three-dimensional device [12].

To investigate the squeeze-film damping, the mechanical model is coupled with the Reynolds equation [13]. The governing system is discretized to get a simplified model in order to calculate the force acting on the beam owing to the pressure of the squeeze gas film.

\section{Problem formulation}

The mechanical equation that describes the transversal deflection $w(x, t)$ of an electrically actuated microbeam modelled within the framework of the strain-gradient elasticity theory and by accounting for the fringing field effects reads [10]:

$$
\begin{gathered}
\rho A \ddot{w}+\hat{c} \dot{w}+D_{1} w^{i v}-D_{2} w^{v i}-E A\left(N_{0}+\frac{1}{2 L} \int_{0}^{L} w^{\prime 2} d x\right) w^{\prime \prime}= \\
=\frac{\varepsilon_{0} \varepsilon_{r} b V^{2}}{2(g-w)^{2}}\left(1+\frac{2}{\pi} \frac{(g-w)}{b}\right)-f_{p}(x, t) .
\end{gathered}
$$

$\rho$ is the mass density and $\hat{c}$ the mechanical damping, $A=$ $b d$ is the cross-section area, being $d$ and $b$ the beam thickness and the width respectively. As shown in Figure 1 the initial gap between the beam of length $L$ and the ground is $g$. The gap is filled by a dielectric medium of relative permittivity $\epsilon_{r}\left(\epsilon_{0}\right.$ is the dielectric constant of the free space). The beam deflection is driven by an applied electric voltage $V$ that is composed by a static and a dynamic part: $V(t)=V_{d c}+V_{a c}(t)$.

The parameters $D_{1,2}$ in Eqn. (1) are respectively defined as:

$$
D_{1}=E I+\mu A\left(2 l_{0}^{2}+\frac{8}{15} l_{1}^{2}+l_{2}^{2}\right), \quad D_{2}=\mu I\left(2 l_{0}^{2}+\frac{4}{5} l_{1}^{2}\right) ;
$$

$\mu$ is the Lamé's second parameter. $l_{n}(n=0 ; 1 ; 2)$ are the high-order material length parameters introduced by the non-classical formulation [11]. In a classical formulation $D_{2}=0$ and $D_{1}$ reduces to the bending stiffness $E I$, where $E$ and $I$ are the Young modulus and the moment of inertia, respectively.

The term $f_{p}(x, t)$ in Eqn. (1) represents the force due to the pressure field of the squeeze gas film trapped between the moving microbeam and the substrate. Indicating with $\bar{p}(x, y, t)$ the absolute pressure and with $p_{0}$ the ambient pressure:

$$
f_{p}(x, t)=\int_{0}^{b}\left(\bar{p}(x, y, t)-p_{0}\right) d y .
$$


The suitable boundary conditions for a calmped-clamped beam are:

$$
w=w^{\prime}=w^{\prime \prime}=0 \quad \text { at } \quad x=0, L
$$

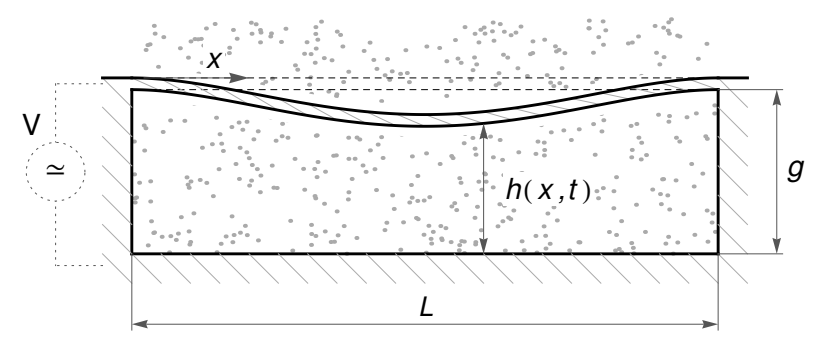

Fig. 1. A schematic drawing of the fully-clamped electricallyactuated microbeam.

The behavior of the fluid is governed by Reynolds equation [13], due to small dimensions in microstrucures the inertial effect is often negligible and the dynamics of the film between two surfaces in relative motion can be described by

$$
\frac{\partial}{\partial x}\left(h^{3} \bar{p} \frac{\partial \bar{p}}{\partial x}\right)+\frac{\partial}{\partial y}\left(h^{3} \bar{p} \frac{\partial \bar{p}}{\partial y}\right)=12 \eta\left(h \frac{\partial \bar{p}}{\partial t}+\bar{p} \frac{\partial h}{\partial t}\right),
$$

where $h(x, t)=g-w(x, t)$. The boundary conditions for the fluid are:

$$
\begin{aligned}
& \bar{p}=p_{0} \quad \text { at } \quad y=0, b, \\
& \frac{\partial \bar{p}}{\partial x}=0 \quad \text { at } \quad x=0, L .
\end{aligned}
$$

\subsection{The dimensionless problem}

Introducing the following dimensionless variables (starred ones):

$$
w^{*}=\frac{w}{g}, \bar{p}^{*}=\frac{\bar{p}}{p_{0}}, h^{*}=\frac{h}{g}, x^{*}=\frac{x}{L}, y^{*}=\frac{y}{b}, t^{*}=\sqrt{\frac{D_{1}}{\rho A L^{4}}} t,
$$

the dimensionless form of the Eqns. $(1,5)$ are respectively

$$
\begin{gathered}
\frac{\partial^{4} w^{*}}{\partial x^{* 4}}-\alpha_{3} \frac{\partial^{6} w^{*}}{\partial x^{* 6}}+c \frac{\partial w^{*}}{\partial t^{*}}+\frac{\partial^{2} w^{*}}{\partial t^{* 2}}=\left(N+\alpha_{1} \int_{0}^{1}\left(\frac{\partial w^{*}}{\partial x^{*}}\right)^{2} d x^{*}\right) \times \\
\frac{\partial^{2} w^{*}}{\partial x^{* 2}}+\frac{\alpha_{2} V^{2}}{\left(1-w^{*}\right)^{2}}\left(1+\beta\left(1-w^{*}\right)\right)-p_{n} \int_{0}^{1}\left(\bar{p}^{*}-1\right) d y^{*}
\end{gathered}
$$

and

$$
\frac{\partial}{\partial x^{*}}\left(h^{* 3} \bar{p}^{*} \frac{\partial \bar{p}^{*}}{\partial x^{*}}\right)+\mathcal{L}^{2} \frac{\partial}{\partial y^{*}}\left(h^{* 3} \bar{p}^{*} \frac{\partial \bar{p}^{*}}{\partial y^{*}}\right)=\tilde{\sigma}\left(h^{*} \frac{\partial \bar{p}^{*}}{\partial t^{*}}+\bar{p}^{*} \frac{\partial h^{*}}{\partial t^{*}}\right) .
$$

The dimensionless boundary conditions are

$$
\begin{gathered}
w^{*}=\frac{\partial w^{*}}{\partial x^{*}}=\frac{\partial^{2} w^{*}}{\partial x^{* 2}}=0, \quad \frac{\partial \bar{p}^{*}}{\partial x^{*}}=0 \quad \text { at } \quad x^{*}=0,1 \\
\bar{p}^{*}=1 \quad \text { at } \quad y^{*}=0,1 .
\end{gathered}
$$

The dimensionless constants and parameters in Eqns. (9, 10) are defined as follows:

$$
\begin{gathered}
\alpha_{1}=\frac{E A g^{2}}{2 D_{1}}, \alpha_{3}=\frac{D_{2}}{D_{1} L^{2}}, c=\frac{\hat{c} L^{4}}{\sqrt{\rho A L^{4} D_{1}}}, \beta=\frac{2}{\pi} \frac{g}{b}, N=\frac{E A N_{0} L^{2}}{D_{1}} \\
\alpha_{2} V^{2}=\frac{b L^{4} \varepsilon}{2 D_{1} g^{3}} V^{2}, \tilde{\sigma}=12 \eta \sqrt{\frac{D_{1}}{\rho A}} \frac{1}{p_{0} g}, \mathcal{L}=\frac{L}{b}, p_{n}=\frac{L^{4} b p_{0}}{D_{1} g},
\end{gathered}
$$

For the sake of simplicity, in the following sections we work with dimensionless variables dropping stars.

\section{Undamped linear mode shapes}

In order to derive the eigenvalue problem associate to Eqn. (9), we impose

$$
w(x, t)=\phi_{n}(x) e^{i \omega_{n} t},
$$

dropping the nonlinear, forcing, and damping terms, we have:

$$
\phi_{n}^{i v}-\alpha_{3} \phi_{n}^{v i}-N \phi_{n}^{\prime \prime}=\omega_{n}^{2} \phi_{n}
$$

with boundary conditions at both edges given by

$$
\phi_{n}=\phi_{n}^{\prime}=\phi_{n}^{\prime \prime}=0
$$

The components of the eigenpair $\left\{\phi_{n}, \omega_{n}\right\}$ are the nth vibrating mode and its own natural (circular) frequency.
(8) 50
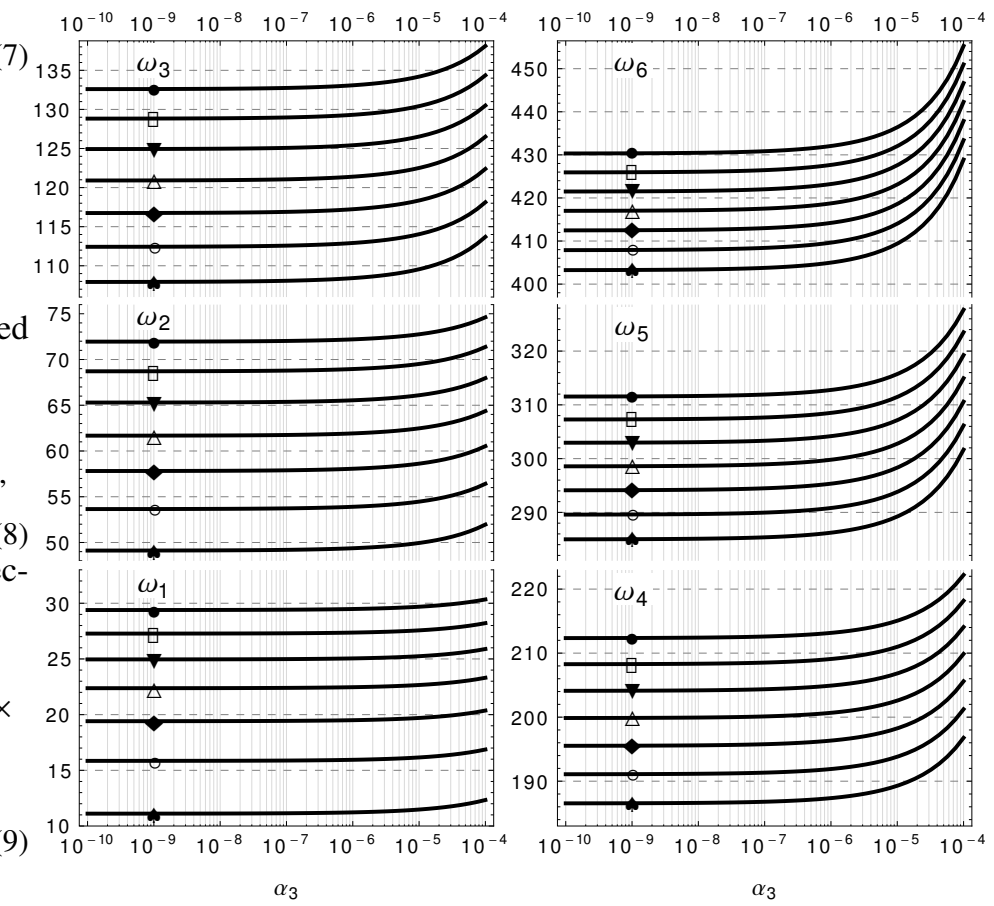

Fig. 2. $N=30(\bullet), N=20(\square), N=10(\boldsymbol{\nabla}), N=0(\triangle), N=-10$ $(\downarrow), N=-20($ ( ),$N=-30($ ()

The variation of the first six eigenfrequencies with respect to $\alpha_{3}$ for different values of axial load is shown in Figure 2. The plot shows how the influence is reduced decreasing the value of $\alpha_{3}$. It can be seen that the higher eigenfrequencies are more affected by $\alpha_{3}$. 


\section{Mathematical model and the approximate solution}

We look for a solution of Eqn. (9) that is the sum of a static and dynamic response of the system:

$$
w(x, t)=w_{s}(x)+w_{d}(x, t) .
$$

Under the hypothesis of small oscillations around an imposed static position, the variation of the pressure can be considered small too, thus

$$
\bar{p}(x, y, t)=1+p(x, y, t) .
$$

With such a decomposition the mechanical and fluid-dynamical equation became respectively

$$
\begin{gathered}
w_{s}^{i v}+w_{d}^{i v}-\alpha_{3} w_{s}^{v i}-\alpha_{3} w_{d}^{v i}+c \dot{w}_{d}+\ddot{w}_{d}= \\
=\left[N+\alpha_{1} \int_{0}^{1}\left(w_{s}^{\prime 2}+2 w_{s}^{\prime} w_{d}^{\prime}+w_{d}^{\prime 2}\right) d x\right]\left(w_{s}^{\prime \prime}+w_{d}^{\prime \prime}\right)+ \\
+\frac{\alpha_{2}\left(V_{d c}^{2}+2 V_{d c} V_{a c}+V_{a c}^{2}\right)}{\left(1-w_{s}\right)^{2}\left(1-\frac{w_{d}}{\left(1-w_{s}\right)}\right)^{2}}\left(1+\beta\left(1-w_{s}-w_{d}\right)\right)-p_{n} \int_{0}^{1} p d y,
\end{gathered}
$$

and

$$
\begin{gathered}
\frac{\partial}{\partial x}\left(\left(1-w_{s}-w_{d}\right)^{3} \bar{p} \frac{\partial \bar{p}}{\partial x}\right)+\mathcal{L}^{2} \frac{\partial}{\partial y}\left(\left(1-w_{s}-w_{d}\right)^{3}(1+p) \frac{\partial p}{\partial y}\right) \\
=\tilde{\sigma}\left(\left(1-w_{s}-w_{d}\right) \frac{\partial p}{\partial t}-(1+p) \frac{\partial w_{d}}{\partial t}\right) .
\end{gathered}
$$

\subsection{The static solution}

Getting rid of the dynamical dependences in Eq. (19) leads to the static problem

$w_{s}^{i v}-\alpha_{3} w_{s}^{v i}-\left[N+\alpha_{1} \int_{0}^{1} w_{s}^{\prime 2} d x\right] w_{s}^{\prime \prime}=\frac{\alpha_{2} V_{d c}^{2}}{\left(1-w_{s}\right)^{2}}\left(1+\beta\left(1-w_{s}\right)\right)$.

with

$$
w_{s}=w_{s}^{\prime}=w_{s}^{\prime \prime}=0 \quad \text { at } \quad x=0,1 .
$$

We express the solution of Eq. (21) as

$$
w_{s}(x)=\sum_{i}^{N} a_{i} \phi_{i}(x) .
$$

The shape functions used in the sum are the solutions of eigenvalue problem (15), the coefficients $a_{i}$ are to be determined by means of the well-known Galerkin procedure.

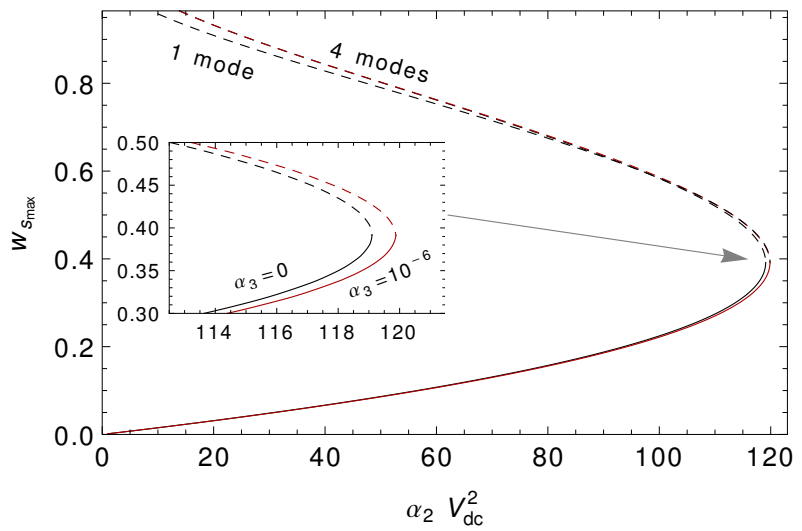

Fig. 3. The static solution. Stable branch (solid lines), unstable branch (dashed line). $\alpha_{1}=5.63, N=30$. The black line refers to a microbeam with $\alpha_{3}=0$ while the red one has $\alpha_{3}=10^{-6}$
Due to the symmetry of the problem, only the odd modes are here considered, this assumption is confirmed by the numerical results on the coefficients of Eq. (23).

In Figure 3 is shown the maximum static deflection $w_{s_{\max }}=w_{s}(x=0.5)$ as function of $\alpha_{2} V_{d c}^{2}$. It is reported the behaviour towards the static pull-in for two microbeams with $\alpha_{3}=0$ and $\alpha_{3}=10^{-7}$. Increasing the effect of the high-order material parameters, the static instability limit moves towards high values of $\alpha_{2} V_{d c}^{2}$. With dashed lines are reported the unstable branches of the curves. We reported in the same figure the convergence of the solution using one or four modes in Eqn. (23). A sensible difference is visible only for the last part of the unstable branches.

\subsection{The linearized governing equations}

The linearization of Eqn. (20) to the first order in $p$ and $w_{d}$, gives:

$$
\begin{aligned}
\left(1-w_{s}\right)^{3} \frac{\partial^{2} p}{\partial x^{2}} & -3\left(1-w_{s}\right)^{2} \frac{\partial w_{s}}{\partial x} \frac{\partial p}{\partial x}+\mathcal{L}^{2}\left(1-w_{s}\right)^{3} \frac{\partial^{2} p}{\partial y^{2}} \\
& =\tilde{\sigma}\left(\left(1-w_{s}\right) \frac{\partial p}{\partial t}-\frac{\partial w_{d}}{\partial t}\right) .
\end{aligned}
$$

The boundary conditions are:

$$
p=0 \quad \text { at } \quad y=0,1
$$

and

$$
\frac{\partial p}{\partial x}=0 \quad \text { at } \quad x=0,1 .
$$

We recast the Eqn. (19) expanding in Taylor series around $w_{s}$ the forcing term. In the analysis we do not consider the mechanical damping and as usual $V_{a c}^{2}$ can be neglected; furthermore using Eqn. (21) we get:

$$
\begin{gathered}
w_{d}^{i v}-\alpha_{3} w_{d}^{v i}-N w_{d}^{\prime \prime}+\ddot{w}_{d}=\left[\alpha_{1} \int_{0}^{1}\left(2 w_{s}^{\prime} w_{d}^{\prime}\right) d x\right] w_{s}^{\prime \prime}+ \\
{\left[\alpha_{1} \int_{0}^{1}\left(w_{s}^{\prime 2}+2 w_{s}^{\prime} w_{d}^{\prime}\right) d x\right] w_{d}^{\prime \prime}+\alpha_{2} V_{d c}^{2} w_{d}\left(\frac{2+\beta\left(1-w_{s}\right)}{\left(1-w_{s}\right)^{3}}\right)+} \\
2 \alpha_{2} V_{d c} V_{a c}\left(\frac{1-\beta\left(1-w_{s}\right)}{\left(1-w_{s}\right)^{2}}\right)-p_{n} \int_{0}^{1} p d y
\end{gathered}
$$

The boundary conditions read

$$
w_{d}=w_{d}^{\prime}=w_{d}^{\prime \prime}=0 \quad \text { at } \quad x=0,1 .
$$

\section{The squeeze-film damping model}

In this section we follow the model proposed by [8] to solve the coupled equations of Sect. 4.2. We express the harmonic excitation and the unknown functions as follows:

$$
\begin{gathered}
p(x, y, t)=\sum_{m} b_{m} \cos (m \pi x) \sin (\pi y) e^{i \lambda t} \\
w_{d}(x, t)=A \phi_{1}(x) e^{i \lambda t} \\
V_{a c}=v_{a c} e^{i \lambda t} .
\end{gathered}
$$

Inserting these equations in Eqn. (24) and integrating along the width of the beam, we obtain:

$$
\sum_{m} B_{m} C_{m}(x)=\phi_{1}
$$

being $B_{m}=b_{m} / A$ and

$$
\begin{gathered}
C_{m}(x)=\left\{\left[\frac{\left(1-w_{s}\right)^{3}}{i \sigma}\left((m \pi)^{2}-\mathcal{L}^{2} \pi^{2}\right)+\left(1-w_{s}\right)\right] \times\right. \\
\left.\cos (m \pi x)-3\left(\frac{\left(1-w_{s}\right)^{2}}{i \sigma} \frac{\partial w_{s}}{\partial x} m \pi\right) \sin (m \pi x)\right\} \frac{2}{\pi} .
\end{gathered}
$$


We remark that $w_{s}=\sum_{i=1, \text { odd }}^{7} a_{i} \phi_{i}$ and $\sigma=\tilde{\sigma} \lambda$ is the so called squeeze number. Multiplying by $\cos (m \pi x)$ the Eqn. (30) and integrating along $x$ from 0 to 1 , in matrix form we have:

$$
[D]\{B\}=\{c\},
$$

with components described by

$$
\begin{aligned}
c_{m} & =\int_{0}^{1} \phi_{1}(x) \cos (m \pi x) d x, \\
D_{m, n} & =\int_{0}^{1} C_{m}(x) \cos (n \pi x) d x ;
\end{aligned}
$$

for $m, n=1, \ldots, M$.

Solving the algebraic system we can obtain the nondimensional spring and damping force acting on the beam surface, given respectively by:

$$
f_{s}=\frac{2}{\pi} p_{n} \mathfrak{R}(B) \cdot c \quad f_{d}=\frac{2}{\pi} p_{n} \mathfrak{J}(B) \cdot c
$$

From (27), recalling that the eigenfunctions are normalized such that $\int_{0}^{1} \phi^{2} d x=1$, we get the amplitude $A$ :

$$
\begin{gathered}
A=2 \alpha_{2} V_{d c} v_{a c} \int_{0}^{1} \frac{1-\beta\left(1-w_{s}\right)}{\left(1-w_{s}\right)^{2}} \phi_{1} d x \times \\
{\left[\omega_{1}^{2}-\lambda^{2}-\alpha_{2} V_{d c}^{2} \int_{0}^{1} \frac{2+\beta\left(1-w_{s}\right)}{\left(1-w_{s}\right)^{3}} \phi_{1}^{2} d x-2 \alpha_{1} \int_{0}^{1} w_{s}^{\prime} \phi_{1}^{\prime} d x \times\right.} \\
\left.\int_{0}^{1} w_{s}^{\prime \prime} \phi_{1} d x-\alpha_{1} \int_{0}^{1} w_{s}^{\prime 2} d x \int_{0}^{1} \phi_{1}^{\prime \prime} \phi_{1} d x+p_{n} \frac{2}{\pi} \sum_{m} B_{m} c_{m}\right]^{-1}
\end{gathered}
$$

\section{Results}

The Figures 4 and 5 show the variation of the nondimensional damping and spring force (34) as function of the squeeze-film number. The curves present points of intersection, namely the cut-off points [4]. If $\sigma \lesseqgtr \sigma_{\text {cut-off }}$ the damping is respectively less/equal/larger than the spring force. We remark that we are considering the effect of the high-order parameters and the correction of the electric force. Figure 4 highlights the variation of the behaviour with respect to the parameter $\alpha_{2} V_{d c}^{2}$, thus, keeping fixed the physical and geometrical properties within $\alpha_{2}$, the figure illustrates the variation of the cut-off point increasing the static voltage.



Fig. 4. Nondimensional damping and spring force as function of the squeeze-film number. $\mathcal{L}=20, \alpha_{3}=10^{-6}, \beta=0$.

Figure 5 shows the effect of the correction for the fringing field effect. Only the curves with high values of $\alpha_{2} V_{d c}^{2}$ are significantly affected by $\beta$. Finally we tested different value for the $\alpha_{3}$. Increasing this parameter both the curves of spring and damping force slightly translate to higher values. As happens with the parameter $\beta$ the effect of $\alpha_{3}$ is more important at high voltage, i.e. near the static pull-in.

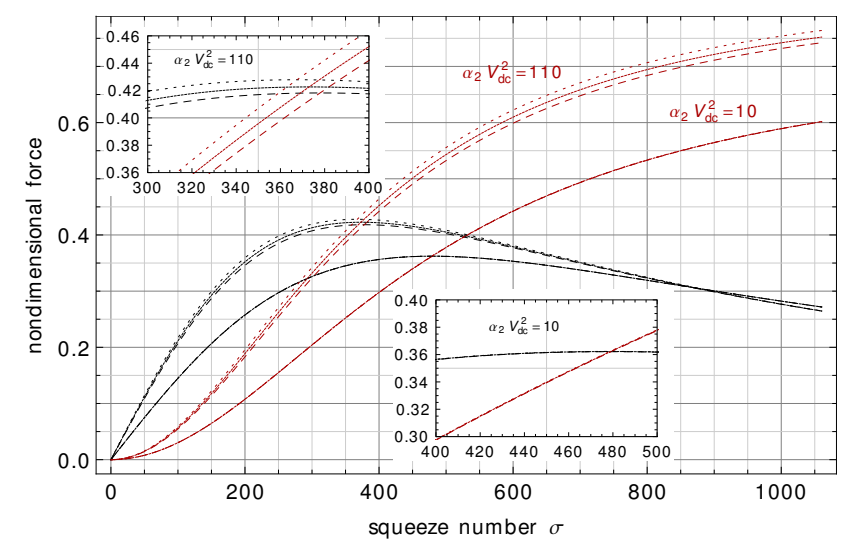

Fig. 5. Nondimensional damping and spring force as function of the squeeze-film number. The dashed, solid and dotted curves refer respectively to $\beta=0, \beta=0.03185$ and $\beta=0.0637$.

\section{Conclusions and further developments}

A parametric analysis to evaluate the squeeze-damping phenomenon on a slender microbeam modelled within the framework of the strain-gradient elasticity theory has been performed. The investigation has accounted for the fringing field effect introducing a correction in the model. Future works will involve further simulations doing a systematic parametric analysis. Other developments are worthy, such comparisons with FE models and extend the analysis to the nonlinear case.

\section{References}

1. H. Luo, G. Zhang, L. Carley, G. Fedder, J. Microelectromech. Syst., 11(3), 188-195 (2002).

2. J.F. Rhoads, S.W. Shaw, K.L. Turner, J. Moehlis, B.E. DeMartini, W. Zhang, J. Sound Vib., 296(45), 797-829 (2006).

3. P. Belardinelli, M. Brocchini, L. Demeio, S. Lenci. Int. J. Eng. Sci. 69, 16-32 (2013).

4. M.I. Younis, MEMS Linear and Nonlinear Statics and Dynamics, Springer, Series: Microsystems, Vol. 20 (2011).

5. T. Veijola, A. Lehtovuori, J. Sound and Vibration 319, 606-621 (2009).

6. M. Bao, H. Yang, Sensors and Actuators A 136, 3-27 (2007).

7. A.H. Nayfeh, M.I. Younis, J. Micromech. Microeng. 14, 170-181 (2007).

8. P. Li, R. Hu, Y. Fang, J. Micromech. Microeng. 17, 1242-1251 (2007).

9. E.M. Abdel Rahman, M.I. Younis, A.H. Nayfeh, A. H., J. Micromech. Microeng. 12(6), 759-766 (2002).

10. P. Belardinelli, S. Lenci, M. Brocchini. ASME J. Comput. Nonlinear Dyn. in press (2013).

11. D. Lam, F. Yang, A. Chong, J. Wang, P. Tong, J. Mech. Phy. Sol., 51(8), 1477-1508 (2003).

12. R.C. Batra, M. Porfiri, D. Spinello, J. Sound and Vibration, 309(35), 600-612 (2008).

13. B.J. Hamrock,Fundamentals of Fluid Film Lubrication, McGraw-Hill (1994). 